\title{
Análises de Dados Diádicos: Um Exemplo a partir da Pesquisa com Casais
}

\author{
Alexsandro Luiz De Andrade ${ }^{1}$ \\ Universidade Federal do Espírito Santo, Vitória, ES, Brasil \\ Vicente Cassepp-Borges \\ Universidade Federal Fluminense, Volta Redonda, RJ, Brasil \\ University of California, Davis, CA, USA \\ Emílio Ferrer \\ University of California, Davis, CA, USA \\ Rozzana Sanchez-Aragón \\ Universidad Nacional Autónoma de México, Ciudad de México, México
}

\begin{abstract}
Resumo
A pesquisa no campo dos fenômenos sociais e psicológicos possui aspectos que são caracterizados pela interdependência de diferentes atores, como o caso das relações de casais. Desta forma, o presente artigo visa apresentar o modelo de análise de dados diádicos a partir de um estudo em contexto de relações amorosas à comunidade científica brasileira. Para tal, um estudo empírico com foco na interação entre aspectos de paixão obsessiva e dimensões de qualidade no relacionamento foi conduzido com 212 casais brasileiros. É dada uma especial ênfase na montagem do banco de dados, que pode ter a estrutura individual, a estrutura diádica ou ainda a estrutura pairwise. Os resultados apresentam um roteiro de orientação dos principais passos da análise diádica, envolvendo também considerações sobre como configurações de paixão e qualidade se relacionam entre parceiros envolvidos em relações amorosas. Embora não se limitando a esta temática, o modelo de análise diádicas é uma importante ferramenta para o estudo dos relacionamentos românticos.
\end{abstract}

Palavras-chaves: Relações amorosas, satisfação com o relacionamento, análise de dados diádicos.

\section{Dyadic Data Analysis: An Example from Couples Research}

\begin{abstract}
Research in the field of social and psychological phenomena has aspects that are characterized by interdependence of different actors, such as the case of the relationships of couples. The aim of this article is to present the dyadic data analysis model from a study in the context of loving relationships to Brazilian scientific community. An empirical study, focusing on the interaction between aspects of obsessive passion and quality dimensions in the relationship, was performed with 212 Brazilian couples.
\end{abstract}

Endereço para correspondência: Universidade Federal do Espírito Santo, Programa de Pós-Graduação em Psicologia, Av. Fernando Ferrari, 514, Goiabeiras, Vitória, ES, Brasil 29075-910. Fone: 27 4009 2504. E-mail: alexsandro.deandrade@yahoo.com. Website: www.labamp.com.br

Apoio: Conselho Nacional de Desenvolvimento Científico e Tecnológico (CNPq) e Coordenação de Aperfeiçoamento de Pessoal de Nível Superior (CAPES) com bolsas de pós doutoramento para os dois primeiros autores. 
A particular emphasis is given to the assembly of the database, which may have the individual structure, the dyadic structure or even the pairwise structure. The results show a step by step guidance of the main stages of dyadic analysis. In additional, we presented considerations about passion and qualityfor both members of relationship. Although not limited to this theme, the dyadic analysis model is an important tool for the study of romantic relationships.

Keywords: Love relationships, relationship satisfaction, dyadic data analysis.

\section{Análisis Datos Diádicos: Un Ejemplo desde la Investigación con Parejas}

\section{Resumen}

La investigación en el campo de los fenómenos sociales y psicológicos tiene aspectos que se caracterizan por la interdependencia de los diferentes actores, como el caso de las relaciones de pareja. Por lo tanto, este artículo presenta el modelo de análisis de datos diádicos de un estudio en el contexto de las relaciones amorosas a la comunidad científica brasileña. Para eso, un estudio empírico centrado en la interacción entre los aspectos de la pasión y de calidad en la relación se llevó a cabo con 212 parejas brasileñas. Se da un especial énfasis en el montaje de la base de datos, que puede tener la estructura individual, la estructura diádica o la estructura pairwise. Los resultados presentan un plan de trabajo con los pasos principales de análisis diádico, que implica consideraciones sobre la configuración de la pasión y la calidad se refieren a los individuos involucrados en las relaciones amorosas. Aunque no se limite a esta temática, el modelo de análisis diádico es una importante herramienta para el estudio de las relaciones románticas.

Palabras clave: Relaciones amorosas, calidad con la relación, análisis de datos diádicos.

Os fenômenos que envolvem os indivíduos no contexto social são, em sua grande parte, formados e percebidos com base em interações entre diferentes pessoas (Aranha, 1993). Um exemplo simples para essa assertiva é quando um terapeuta pergunta o quanto seu cliente se sente feliz com o relacionamento com sua companheira. Não questionando agora as diferentes dimensões de análise para avaliação da qualidade ou abordagens da ciência psicológica sobre o tema, percebemos claramente que o julgamento não dependerá exclusivamente da avaliação do indivíduo, mas também de diferentes características de sua parceira e principalmente do padrão de interação entre os envolvidos no relacionamento (por exemplo: habilidades de comunicação, estratégia de resolução de conflitos, repertório e expectativas sexuais, entre outros; Kashy \& Snyder, 1995).

O exemplo que tratamos anteriormente é uma demonstração do quanto a avaliação dos parceiros sobre aspectos do relacionamento depende do comportamento do(a) companheiro(a). $\mathrm{Na}$ mesma situação, temos clareza também da tendência de erro que o terapeuta pode ter ao avaliar o relacionamento apenas pela percepção do seu cliente, não considerando o que pensa, sente e faz a outra parte da relação. No caso da pesquisa científica com casais, podemos obter mesma tendência de erro ou estimativas falseadas, quando tomamos por decisão avaliar fenômenos em contextos de interação a partir de sua unilateralidade, estratégia metodológica largamente usada nos estudos sobre o amor no Brasil (Cassepp-Borges \& De Andrade, 2013; Cassepp-Borges \& Pasquali, 2012; Cassepp-Borges \& Teodoro, 2007; De Andrade \& Garcia, 2012; Hernandez, 2015; Mônego \& Teodoro, 2011).

Procurando uma solução para estas limitações e contribuindo metodologicamente para o incremento das pesquisas no campo dos fenômenos sociais e psicológicos voltadas para fenô- 
menos caracterizados pela interdependência de diferentes atores (Magsamen-Conrad, Checton, Venetis, \& Greene, 2015), passou-se a adotar os procedimentos de análise diádica de dados "dyadic data analysis" (Kenny, Kashu, \& Cook, 2006). Como exemplos de contexto sociais onde observamos emprego de procedimentos de análise diádica temos: (a) pesquisa em contextos de relações amorosas (Armenta-Hurtarte \& Sánchez-Aragón, 2014); (b) estudo em contexto de família, por exemplo percepção de suporte social e características de interação entre irmãos (Bedin \& Sarriera, 2014); (c) pesquisa em saúde, como por exemplo investigações sobre risco de HIV e uso de drogas entre parceiros(as) (Neaigus, et al., 1995); (d) organizações, como o estudo sobre aspectos de satisfação com o trabalho e relacionamento entre colaboradores (Homburg \& Stock, 2004).

A base estatística da análise diádica assenta-se na ideia de que se dois escores oriundos de dois indivíduos de uma díade são dependentes. Estes dois escores são mais similares um ao outro, do que dois escores de outras duas pessoas que não são membros do mesmo relacionamento, aspecto tecnicamente nomeado de não dependência dos dados (Kenny et al., 2006). Sendo assim, existe uma ligação entre os escores dos integrantes de uma mesma díade, por exemplo, um casal A possui diferenças entre si (sujeito 1 diferente de sujeito 2), no entanto essas diferenças são inferiores às diferenças ao se compararem com um casal B (sujeito 3 e sujeito 4). Esse fato torna o procedimento de análise bastante interessante para o campo de pesquisa em fenômenos de relacionamentos interpessoal, mas também pode generalizar-se em aplicações na área da genética (pesquisa com gêmeos ou com par de cromossomos), organizacional (desempenho e interação com chefia), entre outras.

No que concerte a estrutura dos dados diádicos, três procedimentos são comumente empregados (Kenny et al., 2006; Kenny \& Winquist, 2001): (a) standard dyadic design (padrão de díade): mais comum das estruturas de análise diádicas no qual cada indivíduo é representado isoladamente, bem como seu parceiro(a) de díade (ex. casais). (b) social relations model (relacionamento social): estrutura na qual cada parceiro é uma pessoa independente, porém cada parceiro é pareado com apenas uma pessoa (ex. professores com alunos; gerentes com funcionários). (c) one-with many design (padrões múltiplos): padrão definido por regras prévias delineadas pelo objeto de investigação (ex. membros da família de um indivíduo. A Figura 1 explicita esquematicamente os modelos comentados.

\begin{tabular}{|l|c|c|}
\hline padrão de díade & relacionamento social & padrões múltiplos \\
\hline
\end{tabular}

Figura 1. Estruturas diádicas.

\section{Procedimentos de Organização de Dados Diádicos}

Para proceder com análise de dados por procedimentos diádicos, a organização e o registro de informações no banco de dados exigem uma especial disposição das variáveis, sejam elas para tabulação e/ou futuras análises em softwares estatísticos. Dependo do tipo de pergunta de pesquisa e análise a ser realizada, é necessário modificar a configuração do banco de dados, sendo geralmente necessários bancos de dados paralelos para analisar um mesmo conjunto de dados de uma pesquisa (Kenny et al., 2006). 
São três as formas mais comuns de organização dos dados: (a) estrutura individual (sistemas comumente empregados nas pesquisas de survey, o qual temos numa linha as informações sobre cada participante, sequenciando variáveis levantas/mensuradas por coluna); (b) estrutura diádica: formato no qual a díade respondente do questionário ou variável coletada é disposta

Tabela 1

Modelo de Estrutura de Dados para Análise Diádica é uma única linha, sendo transposto para colunas de variáveis as informações do par correspondente da díade e; (c) estrutura pairwise: caracterizada pela combinação entre organização individual e diádica das informações. Neste formato de organização das variáveis, os dados do participante são acompanhado pelos dados do seu par diádico em cada linha. A Tabela 1 apresenta um modelo simplificados das três estruturas:

Estrutura individual

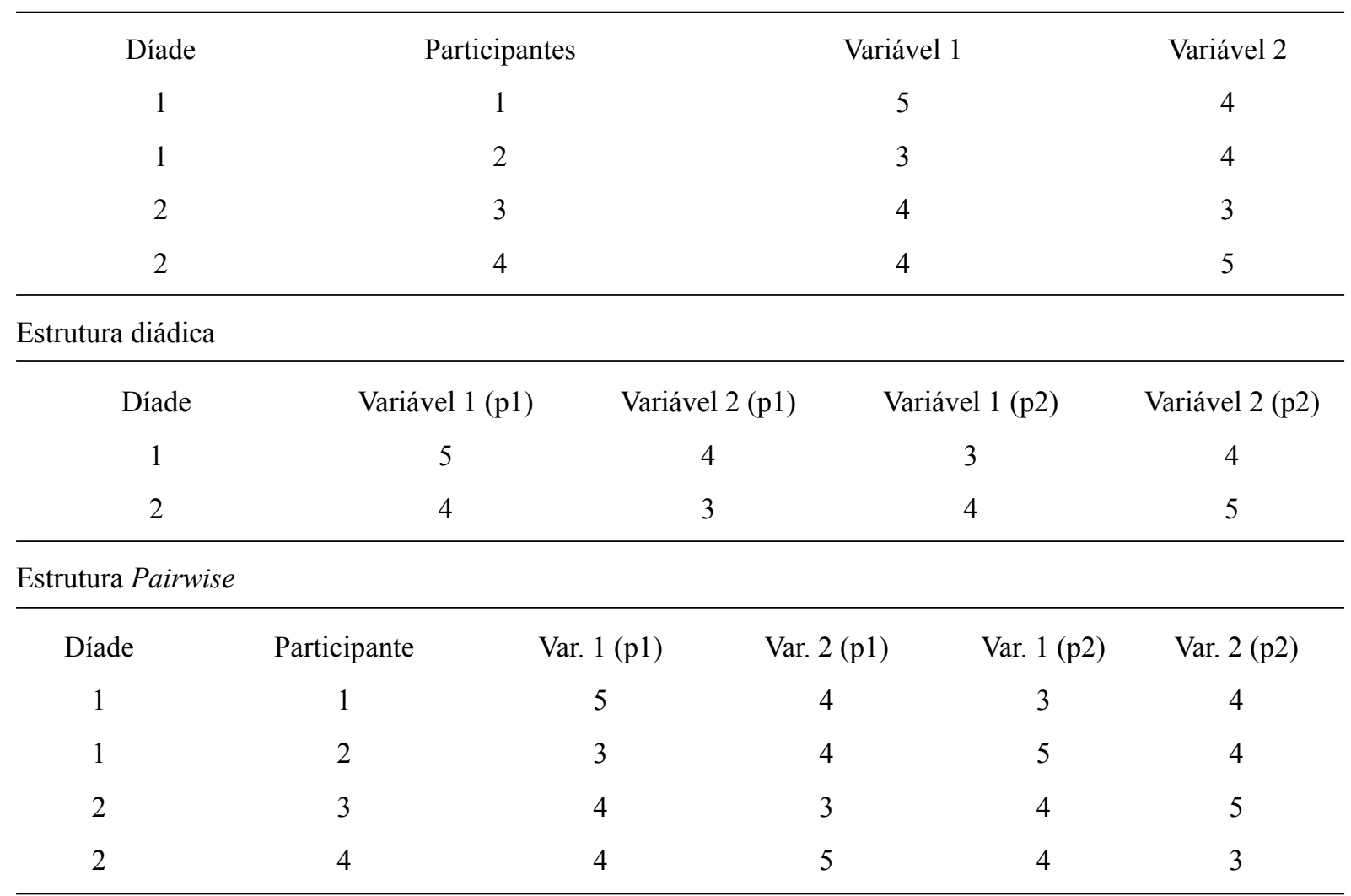

Nota. Os valores atribuídos aos escores de cada participante nas variáveis 1 e 2 consistem em números atribuídos aleatóriamente, apenas para a finalidade de exemplo.

A estrutura individual se aproxima bastante da maneira tradicional de montagem de quaisquer bancos de dados para pesquisas (cada indivíduo apresentado em uma linha, contendo as variáveis pesquisadas nas colunas). No entanto, uma das variáveis deve conter o número da díade à qual o(a) participante pertence (cada díade deve possuir apenas 2 indivíduos). Essa estrutura é sugerida para a realização de análises fatoriais com instrumentos de medida psicológica, testes para a comparação entre os dois membros da dí- ade (por exemplo teste $t$ ), dentre outros (Kenny et al., 2006).

$\mathrm{Na}$ estrutura diádica, cada linha da planilha de organização dos dados não representa mais um sujeito de pesquisa, mas sim uma díade (no caso deste estudo um casal). Desta maneira, a estrutura do banco de dados terá o dobro de variáveis, uma vez que cada variável terá que ser repetida, contendo escores de cada um dos membros da díade, além de metade da amostra total de participantes. A estrutura diádica favorece a 
execução de modelagem por equações estruturais, incluindo path analysis, nas quais variáveis de um dos participantes da díade pode associar-se com as do outro (Kenny et al., 2006).

A estrutura pairwise associa tanto a estrutura individual quanto a estrutura diádica. Nela, cada linha representa um participante de pesquisa. No entanto, as variáveis relativas ao outro membro da díade também são acrescentadas às informações relativas a cada sujeito. É importante observar que cada dado aparece duplicado no banco de registro de informações. Esse tipo de organização de dados é utilizado para análises multinível (Kenny et al., 2006).

Os diferentes métodos da análise diádica vão requerer diferentes formatos em termos de organização do banco de dados. Para análises descritivas, como média e desvio padrão, qualquer uma das três estruturas pode ser usada. No entanto, para alguns procedimentos, apenas determinado tipo de organização, por exemplo: (a) para correlações, sugere-se a organização por díades, a qual pelo menos dentro de uma perspectiva de pesquisa com relacionamento íntimos pode demonstra aproximações e distanciamentos em termos de determinada variável para os dois indivíduos constituintes da díade; (b) para análise do coeficiente inter variáveis "intraclass coefficient", sugere organizações com modelo individual ou pairwise; (c) nas análises de modelagem de equações, incluindo também procedimentos de análise fatorial confirmatória, usa-se comumente o método individual (Ledermann \& Kenny, 2014). De modo geral, o formato dos bancos vai variar segundo objetivo do pesquisado, sendo necessário diante de alguns problemas de pesquisa o uso de 2 ou mais formatos simultaneamente.

Para proceder com estruturação do banco de dados para a análise de díade, o pesquisador pode optar pelo método de cortar e copiar os dados manualmente dentro de um editor de planilha, como MS Excel ${ }^{\circledR}$ ou SPSS ${ }^{\circledR}$. No entanto, soluções mais automátizaveis são encontradas em alguns programas estatísticos. Para tal existe uma sintaxe especifica disponível para o software SPSS (ver Kenny et al., 2006), conjunto de scripts para o software $R$ ( $R$ Development Core
Team, 2011) e aplicativos on line para mudança.

Tanto por critérios de aplicabilidade, como também pela natureza da política de software livre, o pacote "restructure and describe dyadic data (RDDD)" (Kenny, 2015) é uma ferramenta bastante utilizada para reorganizar o banco de dados segundo três tipos de formato (within-dyads, between-dyads, e mixed variables). Como output do programa, obtém-se dois arquivos de dados: (a) arquivo com dados organizados segundo solicitação do operador do software e; (b) arquivo com informações descritivas sobre as variáveis do banco. O programa possui uma interface gráfica de fácil uso e pode ser utilizado com auxílio do pacote RSTUDIO.

Todavia, para usuários não familiarizados com o $R$, o aplicativo disponibilizado on line no site: http://davidakenny.net/, faz este serviço de uma maneira mais intuitiva e prática. Essa opção é de fácil uso, uma vez que não depende da utilização de sintaxes. O primeiro passo é escolher o tipo de estrutura para o qual se quer fazer a transformação (ItoP - Da estrutura individual para a estrutura pairwise; ItoD -Da estrutura individual para a estrutura diádica e; DtoP - Da estrutura diádica para a estrutura pairwise). Depois segue-se fazendo upload da base de dados no website, em formato SPSS (.sav) ou em formato Excel (.csv) e o site retornará a base com nova configuração dos dados em formato .csv. Caso se deseje utilizar o ambiente SPSS para análise seguintes, deve-se fazer novamente a conversão do formato .csv para .sav.

\section{Modelos de Análise Diádica}

Existem inúmeros modelos e avanços no campo da análise diádica. Todavia, consideramos, com base em Kenny et al. (2006), duas opções de grande aplicação na pesquisa psicológicas sobre relações amorosas. São elas o APIM e o modelo de traços latente.

O primeiro modelo, que vem sendo comumente utilizado, é Actor-Partner Interdependence Model - APIM (Cook \& Kenny, 2005). Trata-se de uma modelagem por equações estruturais por meio de análise de caminho ou path analysis (Byrne, 2010), na qual se procura explicar a re- 
lação de uma variável independente (VI) observada e uma variável dependente (VD) também observada. No entanto, existem duas variáveis, e cada variável aparece duas vezes (uma vez para cada um dos pares da díade). Deve ser observado, portanto, o valor da regressão das variáveis independentes para as dependentes no parceiro A (ex. homem), no âmbito de um parceiro provocando mudanças no outro parceiro (ex. qualidade do relacionamento para o homem interferindo na qualidade do relacionamento para mulher), além da correlação entre a variável independente das duas pessoas, e a correlação dos resíduos das duas variáveis dependentes. A estimativa dessas relações facilita a compreensão da influência de uma VI em uma VD, considerando do contexto de uma díade.

A Figura 2 apresenta um exemplo com um diagrama do APIM. De acordo com o exemplo, temos um modelo com quadro variáveis (V1 Homem; V2 Homem; V1 Mulher; V2 Mulher), as quais representam o relacionamento de variáveis coletadas num casal (díade). Assim, temos uma relação entre V1 Homem e V1 Mulher, que poderia ser por exemplo um indicador de paixão correlata do casal. No mesmo modelo, percebemos que as variáveis da díade são mutualmente causadoras de variáveis percebidas individualmente (V2 Homem e V2 mulher). Se tomarmos, por exemplo, V2 como qualidade percebida do relacionamento, temos que a mesma é em parte predita tanto pela paixão do indivíduo (V1 Homem causando V2 Homem; V1 Mulher causando V2 mulher), como também pela variável independente do parceiro (V1 Homem causando V2 Mulher; V1 Mulher causando V2 Homem). Considerando por fim, a relação de interação entre V2 entre ambos os envolvidos na díade.

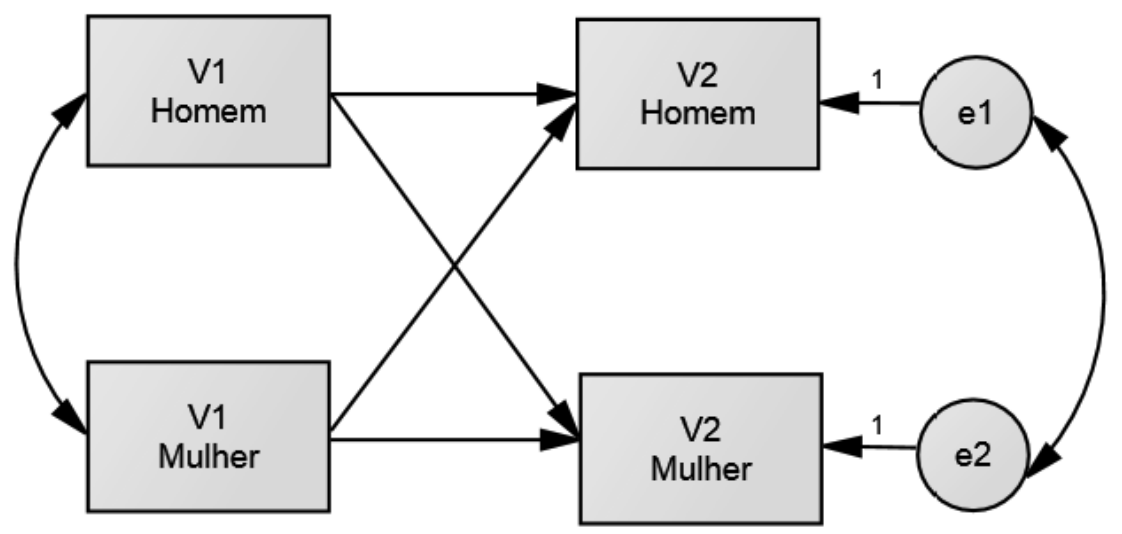

Figura 2. O Actor-Partner Interdependence Model (APIM).

No entanto, o APIM não se trata da única possibilidade para a análise de dados. Um aprimoramento metodológico e teórico é trazer a estrutura de traços latentes para o APIM (Olsen \& Kenny, 2006), perspectiva que permite o uso de estimativas de variáveis não observadas (latentes) e observadas (itens). A vantagem na utilização das variáveis latentes está na possibilidade da criação de um modelo no qual pode-se conhecer as variáveis observadas que estão compondo o traço latente, sua magnitude, bem como conhecer os resíduos de erro associados a cada variável (Byrne, 2010). Quando o traço latente é estimado, ganha-se em precisão, uma vez que no somatório de variáveis para a composição de escores elimina-se a parte de que cada variável observada tem relação com o traço com uma carga diferente, estimando que uma maior precisão do traço reflita em numa maior confiança nos valores de correlações e regressões do modelo.

O presente artigo tem dois objetivos: (a) discutir métodos para a análise de dados diádicos e (b) apresentar um exemplo empírico sobre a perspectiva de análise de dados diádicos no contexto das pesquisas sobre relações amorosas. 
Todavia, destacamos a possibilidade de análise para diferentes fenômenos nos quais o contexto de análise de variáveis de pares se faça interessante. Espera-se que este exemplo possa ampliar os recursos metodológicos e perspectivas sobre o estudo dos relacionamentos interpessoais na ciência brasileira, além de ampliar o conhecimento sobre a especificidade desta forma de análise de dados.

\section{Método}

\section{Participantes}

A amostra do presente estudo contou com 212 casais autodenominados heterossexuais (414 participantes) de diferentes cidades da Região Sudeste (Rio de Janeiro, Minas Gerais e Espírito Santo). A média de idade dos participantes foi de 29,70 anos $(D P=11,60$ anos $)$, com tempo médio de relacionamento de 7,70 anos $(D P=9,71$ anos). O tipo de relacionamento predominante da amostra foi do tipo namoro $(n=257 ; 63,0 \%)$, seguido por casamento $(n=140 ; 34,3 \%)$. Quanto a religião, os participantes em sua maioria eram católicos ( $n=191 ; 46,8 \%)$, seguidos por evangélicos $(n=86 ; 21,1 \%)$ e sem religião mas com crenças em deus $(n=63 ; 115,4 \%)$. Por fim, quanto a variável de ter filho, 26,5\% $(n=108)$ dos participantes declaram ter filhos e $69,9 \%$ ( =285) não.

\section{Instrumentos}

Foi utilizado um formulário autoaplicável, que possuía as seguintes variáveis e escalas psicológicas: (a) Questionário sócio demográfico contendo as informações prévias dos participantes (sexo, idade, tempo de relacionamento, crença religiosa, etc.); (b) A Escala de Avaliação da Qualidade dos Relacionamentos Amorosos (Aquarela-R) - o instrumento original que possui 44 itens bipolares e avaliados por escala de sete intervalos, dispostos numa estrutura multidimensional que avalia aspectos ligados à qualidade e desejabilidade que o relacionamento possui para o indivíduo, diante dos aspectos de comunicação (ex. Imprecisa - Precisa), sexo (ex. Não intenso - Intenso), compromisso (ex. Falso - Verdadeiro), amor (ex. Recíproco - Não recíproco) e intimidade (ex. Carinhosa - Não carinhosa), todas as subescalas possuem indicadores de precisão superiores as 0,90 (De Andrade \& Garcia, 2012); (c) Escala Muldimensional de Paixão (Sánchez Aragón, 2004): instrumento com escala do tipo Likert de 26 itens e quatro dimensões (reações fisiológicas da paixão, paixão persecutória, paixão dependente e desejo-felicidade). A versão adaptada pelos pesquisadores neste estudo apresentou 4 dimensões, sendo três de aspectos negativos da paixão e uma positiva: persecutório (ex. Perseguir "nome do parceiro" é a única maneira de entrar em contato com ele/ ela), dependência (ex. Minha vida é sem sentido sem "nome do parceiro"), reação fisiológica (ex. Eu me sinto ansioso/a quando estou separado/a de "nome do/a parceiro/a"). Todas as dimensões obtiveram indicadores adequados de validade e precisão alfa de Crombach superiores 0,77 . Os dados da dimensão positiva da paixão, ligada a aspectos de desejo-felicidade foram suprimidos das análises por decisão dos pesquisadores em apresentar um modelo mais simples (parcimonioso) neste estudo.

\section{Procedimentos}

A presente pesquisa foi primeira avaliada e obteve parecer favorável do comitê de ética em pesquisa da Universidade vínculo dos pesquisadores (protocolo número: 38697914.8 . 0000.5542 ). O processo de coleta de dados foi conduzido por três assistentes de pesquisa, sendo os participantes recrutados pelo método "bola de neve" (snowball). A aplicação dos questionários ocorreu a partir de convites feitos a casais. Entretanto, os questionários foram aplicados de maneira individual, sendo um questionário para o homem e outro questionário para mulher, ambos contendo os mesmos itens e disposição das perguntas. Durante o processo de aplicação dos questionários, procurou-se isolar os participantes de uma mesma díade, visando a não influência do padrão de respostas entre os mesmos. Em todas as aplicações de questionário, um Termo de Consentimento Livre e Esclarecido com informações sobre a pesquisa foi entregue e lido pelos participantes. Após sua concordância por assinatura, deu-se a entrega do formulário com 
as subescalas do estudo. O tempo de aplicação dos questionários foi em torno de 30 minutos.

\section{Procedimento de Análise dos Dados}

Primeiramente, registrou-se os dados segundo formato participantes e variáveis por linha no software SPSS for Windows ${ }^{\circledR}$. Posteriormente, preparou-se os dados utilizando procedimentos descritivos, além de análise dos fatores para levantamento dos indicadores de validade do construto paixão e precisão dos seus subfatores, os quais resultaram os escores das variáveis do estudo.

Na sequência, para modificação do banco de dados para estrutura de díade, recorreu-se ao aplicativo on line ItoD (disponível em https://davidakenny.shinyapps.io/ItoD/). As análises dos modelos APIM e de traço latente foram feitas por meio de modelagem de equações estruturais. Os dados foram analisados com a utilização do software estatístico MPlus versão 7.2 (Muthén \& Muthén, 2012). Uma parcela das análises foi repedida no software AMOS for Windows ${ }^{\circledR}$, visando uma melhor visualização das informações.

Utilizou-se o método de estimação ML (Maximum Likelihood) para estruturação dos modelos. Foram analisados os seguintes índices de ajuste, de acordo com recomendações de Byrne (2010) e Hair, Black, Babin, Anderson e Tatham (2009):

1. $\chi^{2}$ (qui-quadrado): indicador que avalia a probabilidade do modelo selecionado em se ajustar aos dados da matriz.

2. $\chi^{2} / g l$ : indicador de ajuste no qual recomendam-se valores entre 2 e 3, admitindo-se até 5.

3. CFI (Comparative Fit Index ou índice de ajuste comparativo): é um indicador comparativo referente ao ajuste dos modelos, seus valores variam de 0 a 1 , quanto mais próximos de 1, melhor ajuste, sendo os valores superiores a 0,90 adotados para aceitação do modelo.

4. RMSEA (Root Mean Square Error of Aproximation ou Raíz quadrada media do erro de aproximação): é um indicador de resíduos, sendo recomendados valores situados entre 0,05 a 0,08 , com intervalo de confiança de
$90 \%$, aceitando-se valores de até 0,10 , este índice é mais indicado para estratégias confirmatórias de grandes amostras.

5. GFI (Goodness-of-fit Index ou índice de qualidade do ajuste) e AGFI (Adjusted Goodness-of-fit Index): são indicadores do ajuste ponderado, relacionam-se com proporção de variância-covariância nos dados explicada pelo modelo, com valores variando de 0 a 1 . O valor de parâmetro para aceitação deste é superior ou próximo ao intervalo de 0,90 a 0,95 .

\section{Resultados}

No conjunto das análises diádicas expostas nesse artigo propomos 2 etapas, que são:

1. Registro de dados e estruturação para o modelo de análise diádica e;

2. Testagem dos modelos teóricos. Dentro do teste dos modelos teóricos, pode ser optar por duas alternativas: (a) APIM e; (b) Modelo de traço latente. As etapas descritas a seguir são estruturadas com a finalidade de apresentar um passo a passo, referente aos procedimentos que realizamos no presente estudo, mas que também visam servir de modelo para outros pesquisadores que decidirem pela realização do procedimento de análise com díades.

\section{$1^{\circ}$ Passo: Registro de Dados e Estruturação para o Modelo de Análise Diádica}

Essa etapa de análise, como já mencionado na revisão, pode ser feita diretamente pela tabulação manual dos dados. Todavia, optamos e aconselhamos outros pesquisadores a usarem os aplicativos on line. Dada a natureza deste estudo envolver casais envoltos em um relação romântica estável e o interesse dos pesquisadores em explorar variações entre parceiros do sexo masculino e feminino em termos de aspectos de paixão, qualidade e suas interações, utilizamos um banco de dados com estrutura diádica (standard dyadic design), sendo neste caso a transformação do banco realizada da estrutura individual para a diádica - software ItoD. 
A operação de mudança do banco é visual. Todavia, para que não ocorram confusões, sugere-se o uso de códigos numéricos e alfabéticos fixos para descrição dos participantes (ex. homem, registrar dados usando número 1 e letra $\mathrm{H}$; mulheres, registrar dados usando número 2 e letra M). A Figura 3 apresenta a interface do ItoD no seu site de hospedagem:

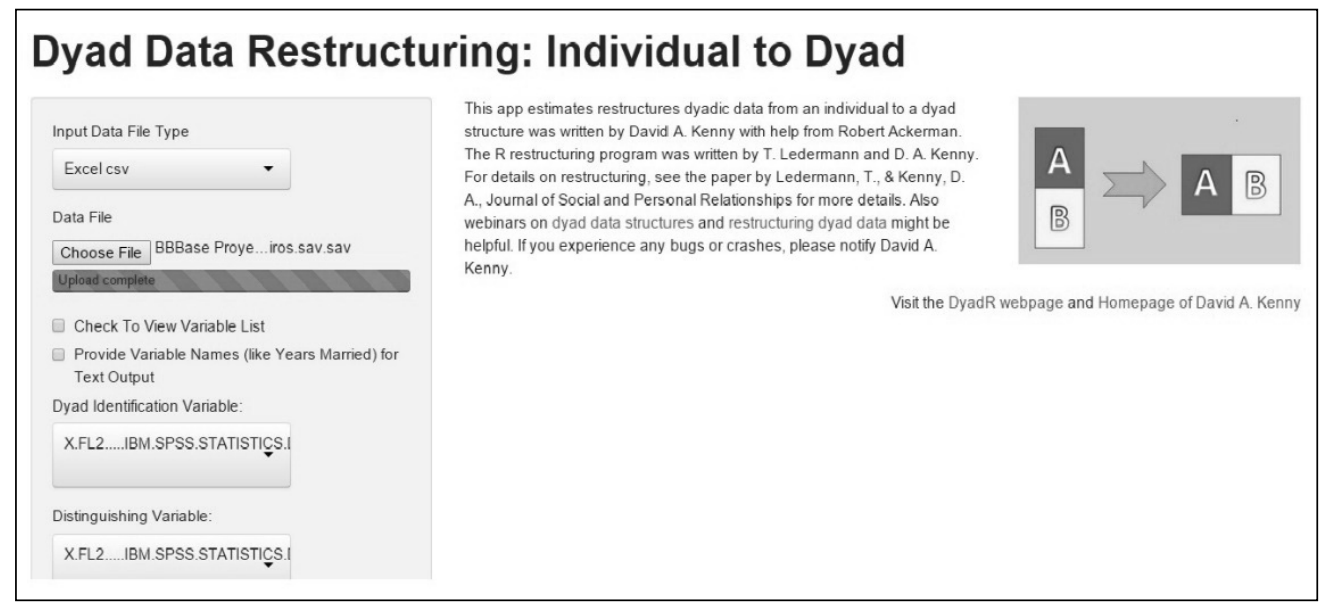

Figura 3. Ambiente de Reestruturação dos dados no ItoD. Fonte: https://davidakenny.shinyapps.io/ItoD/

Com a estruturação de dados realizada de forma exitosa, observa-se que o número de variáveis do banco de dados dobra, da mesma forma que o número de participantes reduz-se na metade. Portanto, na nova estruturação de dados, iremos observar numa mesma linha, ambos os participantes da díade: díade 1, com participante A (por exemplo homem), seguido por variáveis respondidas por $\mathrm{A}$ (dados do questionário dos participantes homens) e na sequência, os dados das participantes $\mathrm{B}$ (dados do questionário das mulheres) ou parceira díade de $\mathrm{A}$.

\section{$2^{\circ}$ Passo: Avaliação do(s) Modelo(s) Teórico(s)}

Dentro do estudo científico dos relacionamentos românticos, um dos temas de maior interesse e importância é a qualidade e/ou satisfação com o relacionamento (Mattson, Rogge, Johnson, Davidson, \& Fincham, 2013). Definida como o julgamento da qualidade do relacionamento a partir de aspectos cognitivos (avaliações), emocionais e comportamentais das interações diádicas dos envolvidos no relacionamento, a avaliação do relacionamento envolve, além de componentes emocionais (ex. amor, paixão, desejo sexual), aspectos cognitivos de atribuição de valia às características do relacionamento (Wachelke, De Andrade, Souza, \& Cruz, 2007). Uma das proposições clássicas sobre qualidade é o modelo de Fletcher, Simpson e Thomas (2000) o qual propõe que aspectos específicos do relacionamento amoroso como intimidade, sexo, comprometimento, comunicação e amor, contribuem com relativa influência para a avaliação global e qualidade do relacionamento. Inúmeros modelos e medidas teóricas se propõem a medir paixão, incluindo o modelo de multifásico de Sánchez Aragón (2004, 2007), no qual a paixão pode ser entendida por ciclos positivos (atração e enamoramento) e negativo (obsessão e desespero).

No segundo passo proposto para análise diádica, associa-se a testagem dos modelos teóricos, podendo este ser estruturado para modelo de variáveis observadas e sem indicação de traços latentes (APIM) ou com modelo de traço latente. A escolha do tipo de procedimento vai depender da natureza dos fenômenos investigados. No caso do presente estudo, a perspectiva tanto de qualidade, quanto paixão encaixam-se adequadamente a modelos de traços latentes, sendo que a proposição teórica escolhida para este estudo busca o entendimento sobre como 
aspectos negativos da paixão se relacionam com aspectos multidimensionais da qualidade do relacionamento.

\section{Análise do Modelo APIM}

O modelo APIM trabalha com os escores composto (variáveis observáveis) ao invés dos modelos traço latente (variável latente e variável observável). A vantagem de utilizar-se esses compósitos de escore está em ter um menor número de parâmetros a serem estimados, facilitando assim a interpretação dos resultados. O
APIM fornece também um excelente indicador da dinâmica da interação entre as variáveis, ainda que o traço latente não seja estimado com precisão (Cook \& Kenny, 2005).

Como exemplo de análise com APIM no presente estudo primeiramente, foram criados escores globais para paixão e qualidade cada membro da díade, optando-se pelo cálculo da média dos itens relativos aos subfatores de cada construto. $\mathrm{Na}$ sequência testou-se o modelo de caminho (path analysis) com auxílio do software AMOS, conforme pode ser observado na Figura 4.

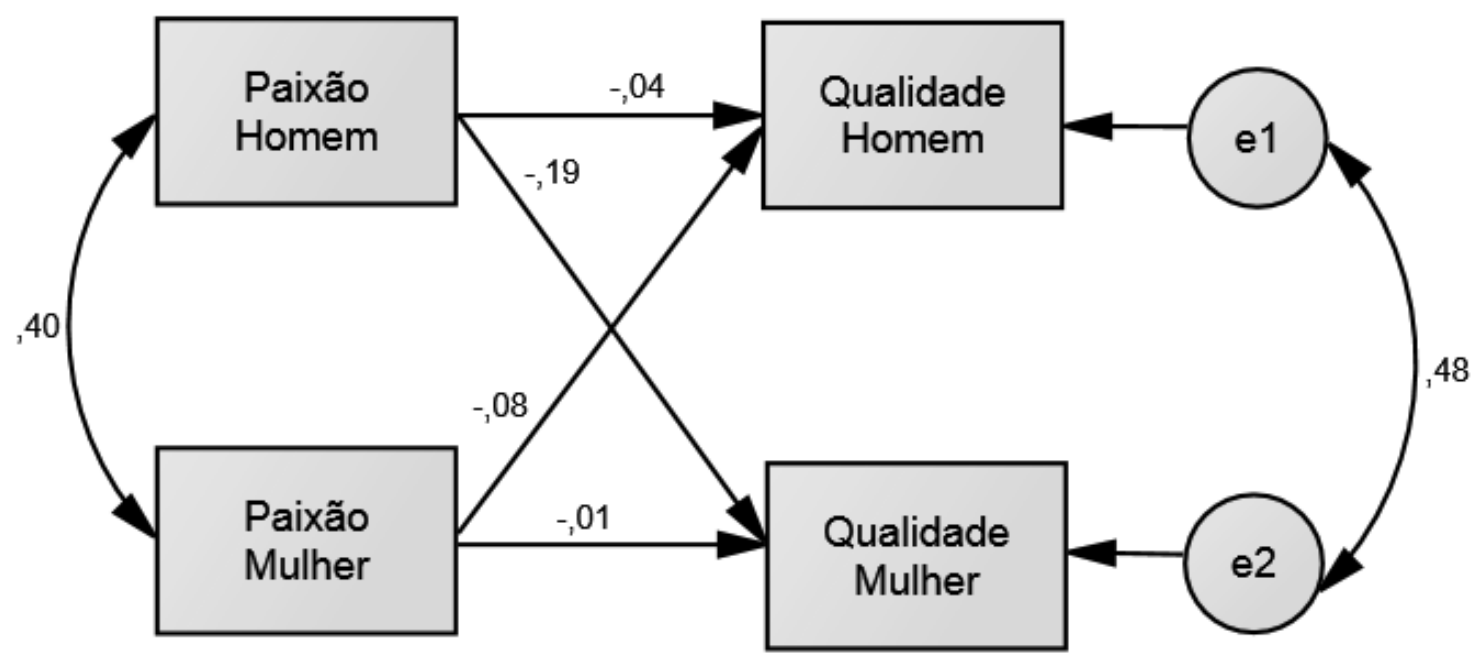

Figura 4. Exemplo empírico do APIM/Paixão e Qualidade.

Os dados do modelo revelam que paixão obsessiva conforme operacionaliza-se neste estudo não prediz qualidade do relacionamento, também se observa uma correlação entre moderada a boa entre paixão obsessiva de homens e mulheres $(0,40)$, bem como de qualidade do relacionamento para os participantes da díade $(0,48$ - no caso do APIM, o resíduo representa a parte das variáveis observadas não explicada pelo restante do modelo. Portanto, a correlação entre os resíduos indica a relação entre as variáveis). Quanto aos indicadores de ajuste do modelo de caminhos, estes não foram plenamente adequados, revelando de algum modo, uma inconsistência do modelo por variáveis observadas: $[\chi 2=$ $139,688, g l=19(p<0,001), \chi 2 / g l=7,35$, RMR $=0,080, \mathrm{GFI}=0,930, \mathrm{AGFI}=0,86, \mathrm{CFI}=0,91$, RMSEA $(90 \% \mathrm{CI})=0,123(0,104-0,142)]$, fato que justifica a busca de um entendimento do fenômeno a partir da perspectiva de traço latente.

Modelo de Traço Latente e Invariância dos Dados

Outra opção para a realização da análise diádica é o teste do modelo de traço latente geral. Este modelo foi construído com estrutura causal semelhante ao APIM apresentado anteriormente, contendo regressões de uma variável masculina influenciando uma feminina e de uma variável feminina influenciando uma masculina. A diferença é que as variáveis observadas com escores gerais foram substituídas pelo traço latente, adotando-se assim uma perspectiva estrutural e multidimensional dos construtos presentes no modelo.

Para avaliação do modelo de traços latente, sugerimos algumas etapas. Deve iniciar-se com: 
(a) à construção do modelo por diagramas de path ou script se a decisão for trabalhar no software R ou Mplus; (b) seu teste na amostra geral; (c) seu teste em amostras específicas por díade (no caso deste estudo estrutura para participantes homens e mulheres) e; (d) modelo referente a interação das variáveis.

Construção do Modelo de Traços Latentes. A construção do modelo de traço latente associa-se ao teste das proposições teóricas do pesquisador. Como técnica base nesta seção da pesquisa, optamos por trabalhar com modelagem de equações estruturais (Byrne, 2010). No caso específico do presente estudo, procurou-se compreender como paixão, interação da paixão do parceiro e interação da qualidade entre os parceiros afetam aspectos multidimensionais da avaliação do relacionamento dos participantes deste estudo.

Para teste de um modelo diádico, são necessárias avaliações intragrupo para ambas as amostras de díade, ou seja, verificar se a estrutura entre variáveis observadas e latentes se mantém semelhante para cada participante da díade. Para tal, prosseguimos com avaliação de 5 modelos: $1^{\circ}$ modelo geral de paixão obsessiva predizendo qualidade multidimensional do relacionamento com todos os participantes do estudo (modelo 1); $2^{\circ}$ modelo específico para primeiro par da díade (no caso deste estudo homens - modelo 2); $3^{\circ}$ modelo específico para o segundo representante da díade (no caso deste estudo mulheres - modelo 3 ); $4^{\circ}$ modelo de interação diádica (incluindo todos os participantes, separados por grupo de variável sexo - modelo 4) e; $5^{\circ}$ modelo de interação diádica com covariância entres os resíduos das variáveis observadas (incluindo todos os participantes, separados por grupo de variável sexo - modelo 5). A Figura 5 representa o diagrama dos 5 modelos testados, bem como seus respectivos índices de ajuste e valores específicos das regressões e correlações do modelo.

No processo de construção dos modelos, observou-se uma evolução na complexidade dos das propostas testadas, as quais culminam com um modelo de interação ajustado. Nesse modelo, percebe-se a preditividade negativa de paixão obsessiva na qualidade do relacionamento do(a) parceiro(a). Ainda que a relação seja fraca é mais impactante para mulheres. Também se destaca a correlação entre paixão obsessiva para homens e mulheres $(0,49)$ e qualidade $(0,51)$, aspecto que sinaliza uma relação de interação moderada à forte entre os aspectos percepto-afetivo dos componentes da díade.

O Teste de Invariância do Modelo de Traço Latente. Caso o pesquisador procure a elaboração de modelos mais parcimoniosos, sugerimos a adoção do teste de invariância do modelo, com base em Ferrer, Balluerka e Widaman (2008) e Widaman, Ferrer e Conger (2010). Tal passo, possibilita verificar de modo mais rigoroso a hipótese de que ambos os integrantes da díade estão respondendo os itens do questionário da mesma forma, em outras palavras, os participantes conseguem ter a mesma interpretação dos itens do questionário. No caso deste estudo com casais, por exemplo, tem-se hipótese a priori de que qualidade e paixão são o mesmo construto tanto para homens quanto para mulheres, fato que se confirma ao final das análises apresentadas.

Analiticamente, para o teste da invariância, segue-se uma avaliação dos modelos com nos seguintes aspectos: (a) estrutura e cargas de fatores e; (b) interceptos e resíduos entre os dois grupos de variáveis (neste caso homens e mulheres). Tal verificação favorece uma maior evidência de que, caso seja encontrada alguma relação significativa entre as regressões dos construtos, ela se refere somente às relações entre os traços latentes (Damásio, 2013) e não a outros aspectos, como por exemplo a compreensão de equivocada de um item dos questionários. No caso de um modelo de traço latente para díades, não há a necessidade (ou mesmo possibilidade) de verificar a hipótese de restrição do número de fatores, comum em testes de invariância de instrumentos de medida.

De maneira a simplificar o modelo apenas, para a realização dos testes de invariância, todas as regressões das modelagens de equações estruturais foram entendidas como correlações. Esse entendimento do modelo foi importante por que, nesse passo, não se buscou o teste da hipótese de interação entre variáveis latentes (o que será 


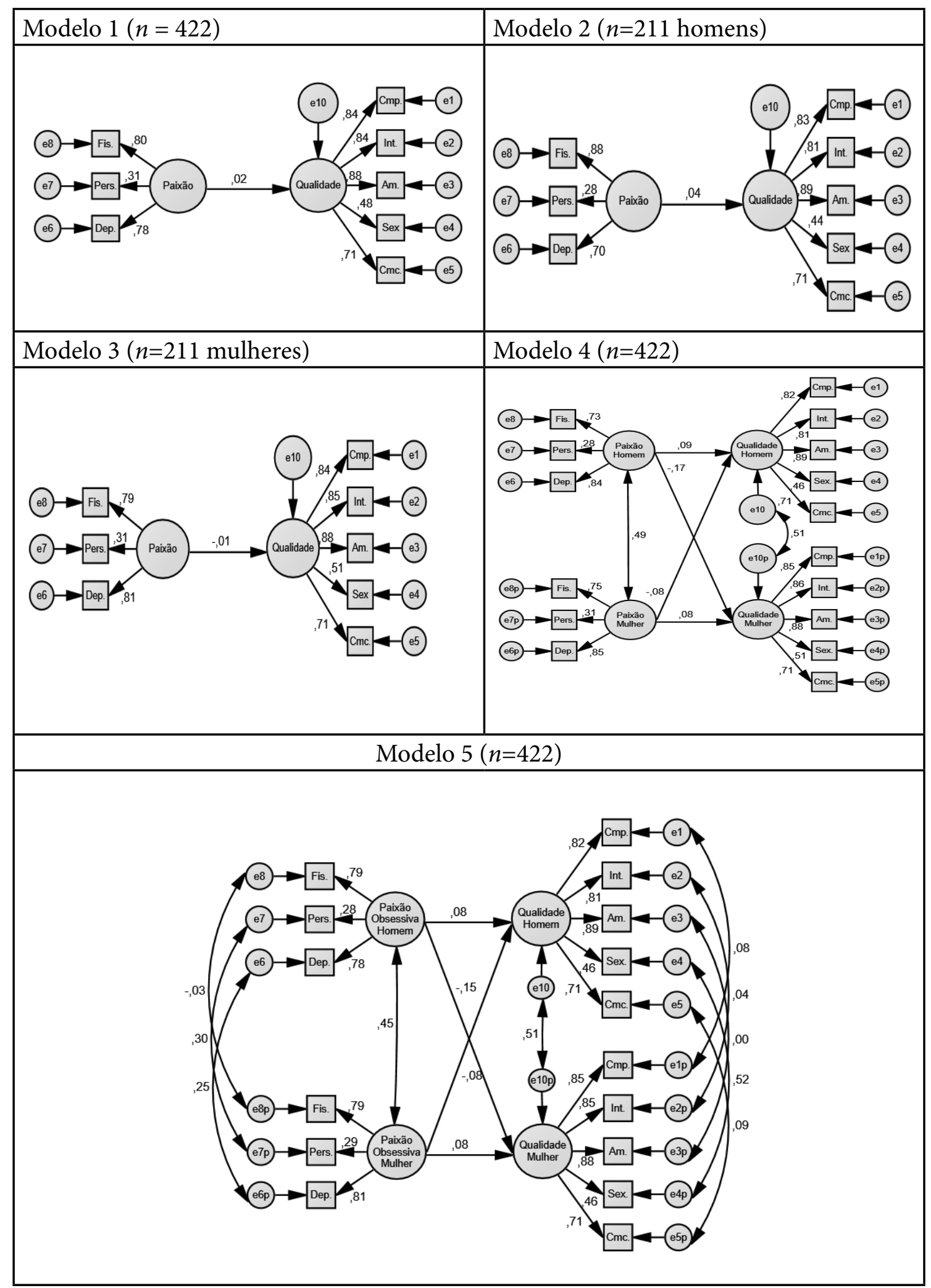

Figura 5. Modelos de Paixão causando Qualidade.

feito no passo seguinte), mas sim o teste da hipótese da invariância. Trabalhar com regressões cria a necessidade de maiores cuidados com o modelo, para que o mesmo seja ajustável (Byr- ne, 2010). O uso das regressões cria dois tipos diferentes tipos de variáveis latentes, de causa e de desfecho. As variáveis de desfecho devem possuir um resíduo associado às mesmas, o que 
as torna diferente das variáveis causadoras (Byrne, 2010; Hair \& et al., 2009). Essa diferença impossibilita a comparação direta dos interceptos, os seja, no caso de uma variável latente de desfecho a comparação é com seu resíduo, não com seu intercepto.

$\mathrm{Na}$ condução do teste da invariância para procedimentos diádicos deste estudo, partimos para o teste de 4 modelos, que são: Modelo 0, Sem restrições (igual ao modelo 5 apresentado na Figura 3, exceto pela utilização de correlações ao invés de regressões); Modelo 1: Restrição das cargas fatoriais, ou seja, as variáveis observadas (itens), possuem os mesmo valores entre homens e mulheres; Modelo 2, restrição dos interceptos, ou seja, a relação entre os traços latentes de paixão e qualidade, é a mesma para homens e mulheres e; Modelo 3: Restrição dos resíduos, ou seja, os resíduos do erro entre todas as variáveis observadas é o mesmo, para homens e mulheres. Para essa etapa da análise, optamos por utilizar o software Mplus, pela sua facilidade em rodar simultaneamente modelagens com estruturas diferentes. A Tabela 2 apresenta os dados de ajuste dos modelos.

Tabela 2

Teste da Invariância do Modelo do Traço Latente

\begin{tabular}{|c|c|c|c|c|c|c|c|c|c|}
\hline Modelo & $\chi^{2}$ & $g l$ & Par.est. & CFI & $\mathrm{BIC}$ & $\begin{array}{l}\text { RMSEA } \\
(90 \% \text { I.C.) }\end{array}$ & $\Delta \chi^{2}$ & $\Delta g l$ & Sig*. \\
\hline Modelo 0 & 212,257 & 90 & 62 & 0,922 & 7627 & $\begin{array}{c}0,080(0,066 \\
-0,094)\end{array}$ & & - & \\
\hline Modelo 1 & 220,969 & 96 & 56 & 0,920 & 7604 & $\begin{array}{c}0,079(0,065 \\
-0,092)\end{array}$ & 8,71 & 6 & $p>0,05$ \\
\hline Modelo 2 & 239,537 & 102 & 50 & 0,912 & 7590 & $\begin{array}{c}0,080(0,067 \\
-0,093)\end{array}$ & 18,57 & 6 & $p<0,05^{*}$ \\
\hline Modelo 3 & 257,370 & 110 & 42 & 0,906 & 7566 & $\begin{array}{c}0,080(0,067 \\
-0,093)\end{array}$ & 17,83 & 8 & $p<0,05^{*}$ \\
\hline
\end{tabular}

Nota. Par.est. (parâmetros estimados), Modelo 0 (Sem restrições); Modelo 1 (Restrição das cargas fatoriais); Modelo 2 (Restrição dos interceptos); Modelo 3 (Restrição dos resíduos).

* Significativo ao nível de $p<0,05$.

Como pode ser observado na Tabela 2, foi demonstrada a invariância apenas no modelo 1 (restrição das cargas fatoriais). A restrição dos interceptos e dos resíduos (modelos 2 e 3), no entanto, aumenta o desajuste do modelo significativamente (indicado pelo qui-quadrado). $\mathrm{O}$ modelo 2, com a restrição dos interceptos, ultrapassou o valor crítico do qui-quadrado, indicando diferenças entre homens e mulheres na maneira em que os itens contribuem para os traços latentes. Quanto ao modelo 3, relativo as restrições de resíduos, também se observam diferenças entre os erros dos participantes dos dois grupos. No entanto, a manutenção do ajuste com a restrição dos resíduos (invariância estrita) é bastante difícil de ser obtida e o desajuste não é estatisticamente significativo ao nível de $p<$ 0,01 . Deve-se atentar ao fato de que o modelo
1 possui importância superior aos demais, pelo fato de que a semelhança entre as cargas fatoriais é o que de diretamente demonstra a semelhança nos traços latentes. Considerando todas as restrições apresentadas nesse parágrafo, é possível entender o modelo como relativamente invariante.

Entendendo o modelo como funcionando da mesma maneira para homens e mulheres, pode-se fazer as interpretações de traço latente propriamente ditas (Byrne, 2010). Para isso, o modelo com graus de liberdade reduzidos é a melhor opção, tendo em vista que os fatores, as cargas, os interceptos e os resíduos não difere significativamente entre homens e mulheres, não é necessário estimar esses valores para cada grupo. É necessário estimar apenas um valor para os dois grupos. Com isso, caso seja encontrada alguma relação entre as variáveis latentes, tem- 
-se uma maior evidência de que de fato existe essa relação entre as variáveis, não se tratando de uma influência de imprecisões na formação destas variáveis latentes (Damásio, 2013).

A Figura 6 representa o diagrama do modelo diádico com controle das invariâncias dos aspectos de fatores e cargas de fatores, interceptos e resíduos, sendo os indicadores de ajuste de: $[\chi 2=257,38, g l=110(p<0,001), \chi 2 / g l=2,33$, SRMR $=0,148, \mathrm{CFI}=0,906$, RMSEA $(90 \% \mathrm{CI})$ $=0,080(0,067-0,093)]$. Note-se que, nesse modelo, novamente estamos trabalhando com regressões ao invés de correlações. Verificada a hipótese da invariância, pode-se retoma-se o objetivo de verificar a influência da paixão sobre a qualidade intra e intersexos.

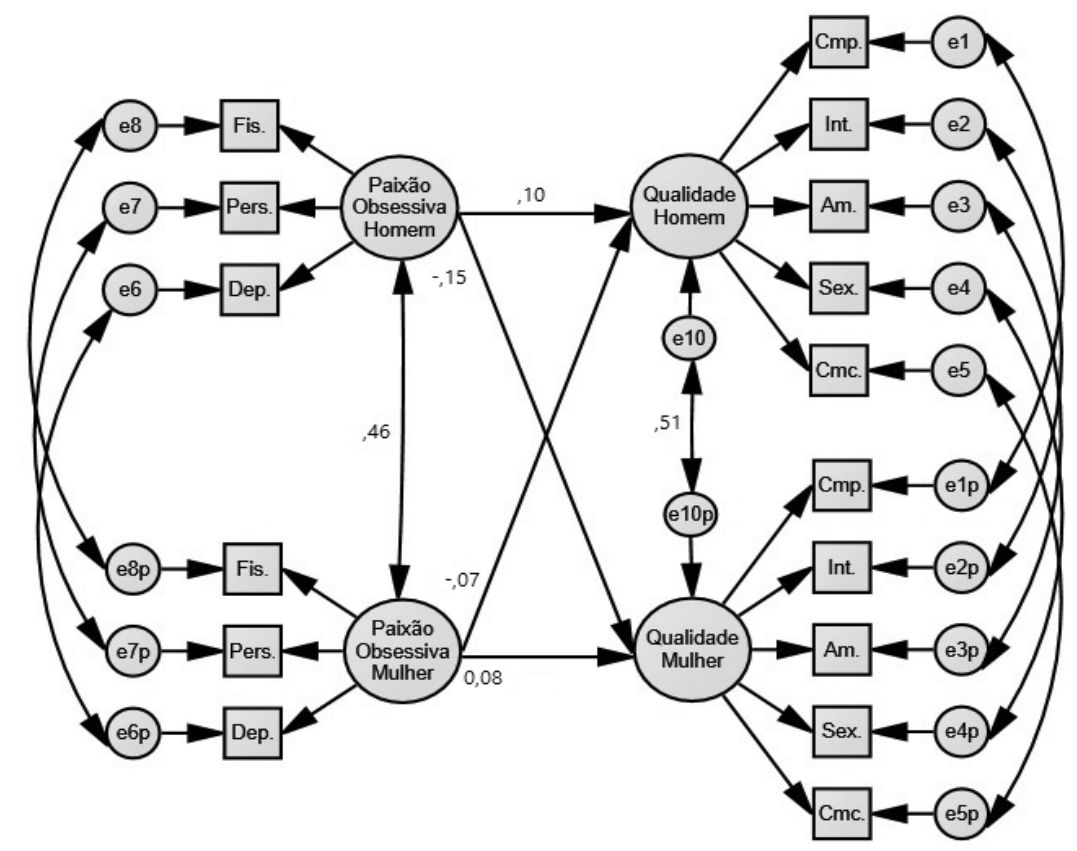

Figura 6. Modelo diádico de paixão e qualidade em casais.

Compreendo o modelo, podemos observar que a correlação entre paixão do homem e da mulher é elevada $(0,45)$, bem como a correlação da qualidade (mensurada pela relação dos erros dos dois traços latentes e igual a 0,51). Como era esperado, paixão, operacionalizada neste estudo por indicadores negativos (dependência e reações fisiológicas) teve baixa predição na qualidade percebida do relacionamento pelo companheiro (comprometimento, intimidade, amor, sexo e comunicação), para mulheres, paixão do parceiro prediz negativamente qualidade $(B=-0,14)$, mesmo vale para homens $(B=-0,06)$, demonstrando que variações emocionais e comportamento de dependência não são adequados para manutenção da qualidade do relacionamentos. Observa-se que a mudança do valor das regressões foi bastante pequena após o cálculo das invariâncias, indicando que o modelo é robusto.

\section{Considerações Finais}

Fenômenos psicológicos não tem sua raiz exclusivamente dentro do próprio indivíduo. Uma díade é um sistema dinâmico, no qual um elemento constantemente influencia o outro (Kenny et al., 2006). Um método de análise que considere essa relação de interdependência representa um enorme ganho na compreensão dos fenômenos diádicos, aspecto almejado na elaboração inicial deste artigo.

Após uma revisão dos diferentes procedimentos de análise adotados, pode-se perceber que características negativas da paixão (obsessão pelo/a parceiro/a) não são um componente favorável para qualidade da relação romântica. $\mathrm{Na}$ mesma direção, evidencia-se que aspectos da percepção dos integrantes do relacionamento são mutualmente relacionados, fato que pode- 
mos observar desde a formulação do APIM até o modelo de traço latente. $\mathrm{O}$ construto paixão obsessiva de homens e mulheres, bem como a qualidade do relacionamento, foram moderadamente correlacionados.

$\mathrm{Na}$ direção dos estudos sobre qualidade em relacionamento realizados em nosso país, De Andrade e Garcia (2012) apontam (não usando procedimentos diádicos de análise) a interdependência dos diferentes aspectos do relacionamento, como por exemplo comunicação, paixão e comprometimento, para avaliações positivas sobre os benefícios do relacionamento romântico. Numa direção metodológica também com estrutura individual Sardinha, Falcone e Ferreira (2009) apontam relações entre empatia do cônjuge, expressão de sentimentos e defesa dos próprios direito com diferentes dimensões da satisfação conjugal. Indicações que corroboram as informações levantadas também neste artigo. Em específico sobre o estudo da paixão, operacionalizada neste estudo apenas pelos indicadores negativos (dependência e reações fisiológicas) do modelo fásico de Sánchez Aragón (2004, 2007), a mesma teve negativa preditividade na qualidade percebida do relacionamento pelo companheiro (comprometimento, intimidade, amor, sexo e comunicação). Para participantes de ambos os sexos, a paixão do parceiro predisse negativamente a qualidade, demonstrando que variações emocionais e comportamentais de dependência não são adequadas para manutenção da qualidade dos relacionamentos.

Do ponto de vista da análise diádica, um dos primeiros desafios deste processo analítico encontra-se na fase de coleta de dados. Ter adesão de participantes para pesquisas no campo das ciências humanos diante da não remuneração, conforme segue o regimento do Ministério da Saúde (2012), é um desafio que vai exigir do pesquisador um grande esforço de planejamento e coleta de dados. Observa-se neste estudo que, quando o processo é demandado para o casal, um investimento maior de tempo e compromisso se faz necessário, incluindo a perda de vários respondentes por um dos parceiros não se interessar em responder o estudo, bem como uma maior atenção e proteção ao sigilo dos dados diante da curiosidade dos participantes em conhecer os comentários do(a) parceiro(a) sobre o relacionamento.

No hall de avanços analíticos para estudo de aspectos interpessoais românticos, existe ainda a limitação do estudo em trabalhar apenas com dados transversais. O procedimento de análise diádica internacionalmente avança para delineamentos com dados longitudinais (Ferrer, Steele, \& Hsieh, 2012), configuração de coleta e análise que, apesar das diferentes dificuldades que possa trazer para o pesquisador, viabiliza considerações teóricas inovadoras para o entendimento do relacionamento romântico.

Modelos de análise de dados diádicos representam um avanço importante na pesquisa em psicologia, destacando-se (e não se limitando ao) estudo dos relacionamentos de casais. A compreensão da interação entre as díades traz uma explicação mais completa do que a simples análise dos elementos individualmente. Espera-se, com esse estudo, que pesquisadores brasileiros compreendam a ferramenta da análise diádica, tanto no nível das variáveis observadas quanto no nível do traço latente, e sejam encorajados a uma maior utilização da mesma.

\section{Referências}

Aranha, M. S. F. (1993). A interação social e o desenvolvimento humano. Temas em Psicologia, 1(3), 19-28.

Armenta-Hurtarte, C., \& Sánchez-Aragón, R. (2014). Dyadic analysis of marital satisfaction: Effects of interaction on the relationship. In A. Garcia (Ed.), Love, Family and Friendship: A Latin American Perspective (pp. 133-143).

Bedin, L. M., \& Sarriera, J. C. (2014). Dyadic analysis of parent-children subjective well-being. Child Indicators Research, 7(3), 613-631. doi:10.1007/s12187-014-9235-9

Byrne, B. M. (2010). Structural equation modeling using AMOS. Basic concepts, applications, and programming. New York: Taylor \& Francis Group.

Cassepp-Borges, V., \& De Andrade, A. L. (2013). Uma breve história das tentativas para medir atributos dos relacionamentos amorosos em língua portuguesa. Estudos de Psicologia 
(Natal), 18(4), 621-628. doi:10.1590/S1413$-294 X 2013000400011$

Cassepp-Borges, V., \& Pasquali, L. (2012). Estudo nacional dos atributos psicométricos da Escala Triangular do Amor de Sternberg. Paidéia (Ribeirão Preto), 22(51), 21-31. doi:10.1590/ S0103-863X2012000100004

Cassepp-Borges, V., \& Teodoro, M. L M. (2007). Propriedades psicométricas da versão brasileira da Escala Triangular do Amor de Sternberg. Psicologia: Reflexão \& Crítica, 20(3), 513-522. doi:10.1590/S0102-79722007000300020

Cook, W. L., \& Kenny, D. A. (2005). The actor-partner interdependence model: A model of bidirectional effects in developmental studies. International Journal of Behavioral Development, 29, 101-109. doi:10.1080/01650250444000405

Damásio, B. (2013). Contribuições da Análise Fatorial Confirmatória Multigrupo (AFCMG) na avaliação de invariância de instrumentos psicométricos. Psico-USF, 18(2), 211-220. doi:10.1590/ S1413-82712013000200005

De Andrade, A. L., \& Garcia, A. (2012). Desenvolvimento de uma medida multidimensional para avaliação de qualidade em relacionamentos românticos-Aquarela-R. Psicologia: Reflexão e Crítica, 25(4), 634-643. doi:10.1590/S010279722012000400002

Ferrer, E., Balluerka, N., \& Widaman, K. F. (2008). Factorial invariance and the specification of second-order latent growth models. Methodology, 4(1), 22-36. doi:10.1027/1614-2241.4.1.22

Ferrer, E., Steele, J. S., \& Hsieh, F. (2012). Analyzing the dynamics of affective dyadic interactions using patterns of intra-and interindividual variability. Multivariate Behavioral Research, 47(1), 136-171. doi:10.1080/00273171.2012.640605

Fletcher, G. J. O., Simpson, J. A., \& Thomas. G. (2000). The measurement of perceived relationship quality components: A confirmatory factor analytic approach. Personality and Social Psychology Bulletin, 26(3), 340-354. doi:10.1177/0146167200265007

Hair, J. F., Black, W. C., Babin, B. J., Anderson, R. E., \& Tatham, R. L. (2009). Análise multivariada de dados. Porto Alegre, RS: Bookman.

Hernandez, J. A. E. (2015). Evidências de validade de construto da Escala de Componentes do Amor. Psicologia: Teoria e Pesquisa, 31(2), 249-257. doi:10.1590/0102-37722015021828249257
Homburg, C., \& Stock, R. M. (2004). The link between salespeople's job satisfaction and customer satisfaction in a business-to-business context: A dyadic analysis. Journal of the Academy of Marketing Science, 32(2), 144-158. doi:10.1177/0092070303261415

Kashy, D. A., \& Snyder, D. K. (1995). Measurement and data analytic issues in couples research. Psychological Assessment, 7(3), 338-348. doi:10.1037/1040-3590.7.3.338

Kenny, D. A. (2015). Restructuring and Describing Dyadic Data (RDDD) Menu. Retrieved from http://davidakenny.net/RDDD.htm

Kenny, D. A., Kashy, D. A., \& Cook, W. L. (2006). Dyadic data analysis. New York: The Guilford Press.

Kenny, D. A., \& Winquist, L. (2001). The measurement of interpersonal sensitivity: Consideration of design, components, and unit of analysis. In J. Hall \& F. Bernieri (Eds.), Interpersonal sensitivity: Theory and measurement (pp. 265-302). Englewood Cliffs, NJ: Erlbaum.

Ledermann, T., \& Kenny, D. A. (2014). A toolbox with programs to restructure and describe dyadic data. Journal of Social and Personal Relationships. doi:10.1177/0265407514555273

Magsamen-Conrad, K., Checton, M. G., Venetis, M. K., \& Greene, K. (2015). Communication efficacy and couples' cancer management: Applying a Dyadic Appraisal Model. Communication Monographs, 82(2), 179-200. doi:10.1080/0363 7751.2014 .971415

Mattson, R. E., Rogge, R. D., Johnson, M. D., Davidson, E. K. B., \& Fincham, F. D. (2013). The positive and negative semantic dimensions of relationship satisfaction. Personal Relationships, 20, 328-355. doi:10.1111/j.14756811.2012.01412.x

Ministério da Saúde. (2012). Resolução $n^{\circ}$ 466, de 12 de dezembro de 2012. Brasília, DF: Autor.

Mônego, B. G., \& Teodoro, M. L. M. (2011). A teoria triangular do amor de Sternberg e o modelo dos cinco grandes fatores. Psico-USF, 16(1), 97105. doi:10.1590/S1413-82712011000100011

Muthén, L. K., \& Muthén, B. O. (2012). MPlus User's Guide ( $7^{\text {th }}$ ed.). Los Angeles, CA: Authors.

Neaigus, A., Friedman, S. R., Goldstein, M., Ildefonso, G., Curtis, R., \& Jose, B. (1995). Using dyadic data for a network analysis of HIV infection 
and risk behaviors among injecting drug users. NIDA Research Monograph, 151, 20-37.

Olsen, J. A., \& Kenny, D. A. (2006). Structural equation modeling with interchangeable dyads. Psychological Methods, 11, 127-141. doi:10.1037/1082-989X.11.2.127

R Development Core Team. (2011). R: A language and environment for statistical computing. Vienna, Austria: R Foundation for Statistical Computing.

Sánchez Aragón, R. (2004). De lo claro a lo oscuro de la pasión. Conferencia presentada en la Semana de la Psicología, Villahermosa, Tabasco, México.

Sánchez Aragón, R. (2007). Pasión romántica: más allá de la intuición, una ciencia del amor. México: Miguel Ángel Porrúa

Sardinha, A., Falcone, E. M. D. O., \& Ferreira, M. C. (2009). As relações entre a satisfação conjugal e as habilidades sociais percebidas no cônjuge. Psicologia: Teoria e Pesquisa, 25(3), 395-402. doi:10.1590/s0102-37722009000300013
Wachelke, J. R., De Andrade, A. L., Souza, A. M., \& Cruz, R. M. (2007). Estudo complementar da validade fatorial da Escala Fatorial de Satisfação em Relacionamento e predição de satisfação global com a relação. Psico-USF, 12(2), 221-225.

Widaman, K. F., Ferrer, E., \& Conger, R. D. (2010). Factorial invariance within longitudinal structural equation models: Measuring the same construct across time. Child Development Perspectives, 4(1), 10-18. doi:10.1111/j.17508606.2009.00110.x
Recebido: $22 / 10 / 2015$

$1^{a}$ revisão: $23 / 06 / 2016$ Aceite final: 07/08/2016 


\section{Anexo}

\section{Escala Multifásica da Paixão para o Português Brasileiro}

Itens

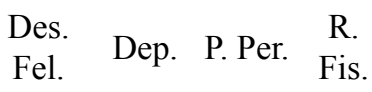

0,92

me faz sentir excitação.

0,86

0,85

0,78

0,77

Paixão09.Eu tenho fantasias com eu espero tocá-lo (a) e ser tocada (o) por ele (a). $\quad 0,74$

Paixão10.Na presença de me faz me sentir apaixonado (a).

0,69

Paixão03. ou pensar em

0,64

Paixão05.Quando estou com eu sou a pessoa mais feliz do mundo.

$0,55 \quad 0,30$

0,50

0,48

Paixão01.Fazer planos com me provoca alegria.

0,38 eu nos completamos.

Paixão20.A vida sem

Paixão23.Para mim, a necessidade de estar perto de é como a necessidade do ar para respirar. para me sentir uma pessoa completa.

Paixão19.Preciso intensamente de não está comigo.

Paixão21.Eu não sei o que fazer se não queira estar comigo, eu faço um esforço para estar

Paixão28.Embora com ele (a).

Paixão27.Eu insisto em ter encontros com embora ele (a) hesite em aceitar

Paixão26.Perseguir é a única maneira de entrar em contato com ele (a).

Paixão24.Eu sinto que não corresponde aos meus sentimentos porque ele (a) ainda não percebeu que ele (a) me ama.

Paixão14.Quando não vejo, com frequência, , não consigo dormir.

Paixão16.Às vezes, não consigo fazer o que é normal na minha vida, porque estou pensando em está todo o tempo.

Paixão13.Não fico quieto (a) se não sei onde

Paixão17.Eu me sinto ansioso (a) quando estou separado (a) de

Número itens

$\begin{array}{llll}12 & 6 & 4 & 4\end{array}$

Alfa Cronbach 